\title{
Existence of Two Phases in Poly(methyl methacrylate)
}

\author{
Kentaro MURAKAMI and Junkichi SOHMA \\ Faculty of Engineering, Hokkaido University, Sapporo 060, Japan.
}

(Received December 25, 1978)

\begin{abstract}
The ESR spectral change in spin-labeled poly(methyl methacrylate) (PMMA) was investigated in the solid phase at various temperatures. There appeared a shoulder on the spectrum from $138^{\circ} \mathrm{C}$ to $188^{\circ} \mathrm{C}$ and it is concluded that this shoulder originates from the existence of the two kinds of motion in this temperature region. It was also found by GPC measurements that the molecular-weight distribution has no relationship to the appearance of this shoulder. The data of the second moments of the PMMA of the ${ }^{1} \mathrm{H}$ NMRspectra indicate that the two kinds of motion were the main chain motions of PMMA. Our results suggest that there is a difference in the aggregation state of PMMA molecules.
\end{abstract}

KEY WORDS ESR / Molecular Motion / PMMA / Aggregation /

The effect of steroregularity on the molecular motion of polymer chains has been reported for poly(methyl methacrylate) (PMMA) by several researchers. ${ }^{1-3}$ The spin label and probe methods have proved useful for obtaining information on the molecular motion of the very rapid mode, $10^{-9} \mathrm{~s}$, which can hardly be detected by other experimental methods in solid polymers. ${ }^{4-8}$ The samples of the spin-labeled PMMA are isotactic-rich in microregularity, since the polymerization of the spin-labeled MMA undergoes an anionic process. We have reported on the analysis of the ESR spectra changes of the spin-labeled $\mathrm{PMMA}^{7}$ but not much attention was given to the effect of the stereoregularity on the molecular motion in our previous reseach. More detailed research was performed on the isotactic-rich samples by using not only ESR but also broad-line NMR. The purpose of this paper is to report on the existence of two phases in the sample; in one phase the molecular motion is quite mobile but similar motion of the molecules is not activated in the other phase at the same temperature.

\section{EXPERIMENTAL}

\section{Sample}

The sample used was poly(methyl methacrylate), which was spin-labeled at the ester side chain, as shown by the following formula.

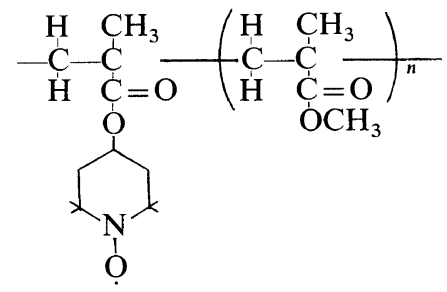

The spin-labeled PMMA was prepared by an anionic copolymerization of MMA monomer and labeled monomer, 4-methacryloyloxyl-2,2,6,6-tetramethylpiperidine, expressed by the following formula, in the presence of a butyllithium catalyst.<smiles>C=C(C)C(=O)OC1CCN(O)CC1</smiles>

The ratio of the nonlabeled side chain to the spinlabeled one was controlled by the mole ratio of MMA monomer and the labeled monomer. The mole ratio of the sample used in this work was taken to be $500: 1$. The tacticity determined by highresolution ${ }^{1} \mathrm{H}$ NMR was isotactic $75 \%$, heterotactic $15 \%$, and syndiotactic $10 \%$ in terms of triads. The molecular weight was determined to be 9500 by the Hitachi 117 V.P.O. molecular weight apparatus. The elution curve of the original sample is shown in Figure 1a. This sample contains the lower- 
molecular-weight fraction, marked with the arrow. The fractionated sample was obtained by using the fractionating GPC, and the elution curve (Figure 1b) demonstrates the removal of the fraction of lower molecular weight.

\section{Spectrometers}

A JEOL PE-X ESR was used with $100 \mathrm{KHz}$ modulation and the temperature control unit attached to the spectrometer was used for temperarature variation experiments (accuracy $\pm 0.5^{\circ} \mathrm{C}$ ). A Varian WL 112 with a ${ }^{1} \mathrm{H}$ resonance frequency of $34 \mathrm{MHz}$ was used for the broad-line NMR measurement.

\section{RESULTS AND ANALYSES OF THE LINE-SHAPES}

\section{ESR Spectra}

The examples of ESR spectra observed from the spin-labeled PMMA in the solid phase are shown in Figure 2 for the various temperatures under observation. The extreme separation between the arrows in Figure 2 is gradually narrowed with raised temperature. This temperature variation in Figure 3 is similar to that reported in the previous paper. ${ }^{7}$ Figure 3 demonstrates that the temperature dependence of the extreme separation is identical for the fractionated and nonfractionated samples. In the spectrum observed at $158^{\circ} \mathrm{C}$ in Figure 2, a shoulder, marked with the asterisk, appears near the central peak. Temperature variations in the line-shape were carefully investigated, as shown in Figure 4, in the temperature range $138-188^{\circ} \mathrm{C}$ in which the shoulder was observed. The positions of both the extreme peak A and the shoulder B from the isotropic $g$ factor $g_{0}$ were plotted against the temperature in Figure 5. Apparently, the position of the extreme peak $\mathrm{A}\left(r_{2}\right)$ gradually changed to $200^{\circ} \mathrm{C}$ and the peak A finally coalesed with the shoulder B.

\section{Determination of Rotational Correlation Times}

When molecular motion is not rapid enough for complete averaging of the anisotropies of the hyperfine and $g$ tensors, one can estimate the rotational correlation time, $\tau_{\mathrm{c}}$, of the nitroxide by the following equation. ${ }^{9}$

$$
\tau_{\mathrm{c}}=c\left(1-\frac{A_{z}}{A_{z}^{*}}\right)^{d}
$$

Where $A_{\mathrm{z}}$ is one-half the separation of the outer hyperfine extreme and $A_{z}{ }^{*}$ is the rigid limit value for the same quanity. The constants $c$ and $d$, are dependent on the different models of diffusion. In this case, the "Moderate Jump Diffusion" model is a reasonable one ${ }^{7}$ and $c$ and $d$ in this model are $1.10 \times 10^{-9} \mathrm{~s}$ and -1.01 , respectively. ${ }^{9}$ The Arrhenius plot of the rotational correlation times is shown in Figure 6. The rotational activation energy is $3.9 \mathrm{kcal} \mathrm{mol}^{-1}$.

$N M R$

The broad-line NMR spectra observed from the

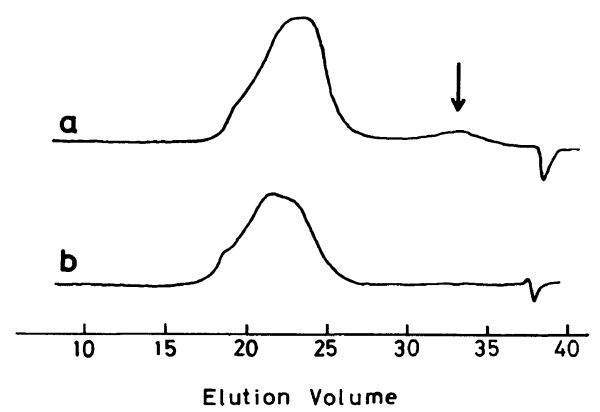

Figure 1. The elution curves of spin-labeled PMMA: a, the original sample; $b$, the fractionated sample.

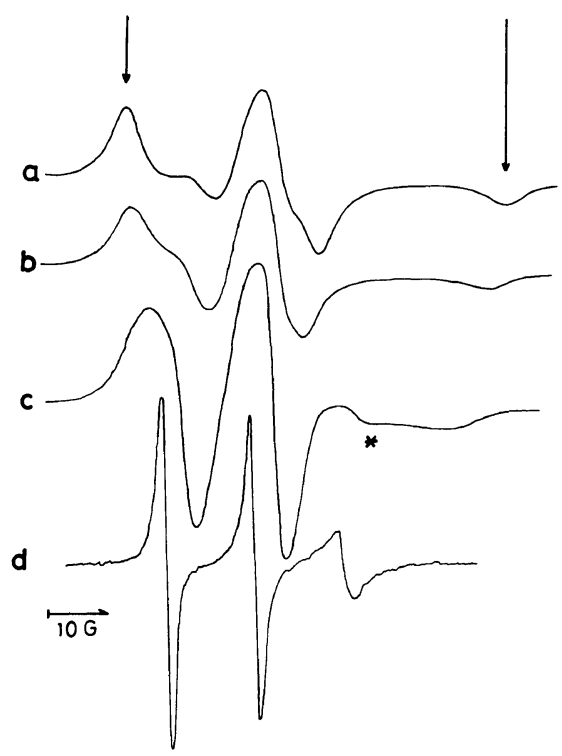

Figure 2. ESR X-band spectra of the spin-labeled PMMA at various temperatures: (a), $52^{\circ} \mathrm{C}$; (b), $119.5^{\circ} \mathrm{C}$; (c), $158^{\circ} \mathrm{C}$; (d), $232^{\circ} \mathrm{C}$. 


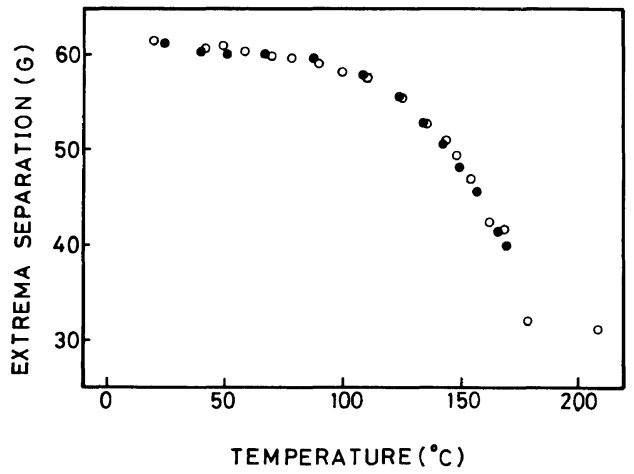

Figure 3. The temperature dependence of the extreme separation: $\bigcirc$, the original sample; $\boldsymbol{O}$, the fractionated sample.

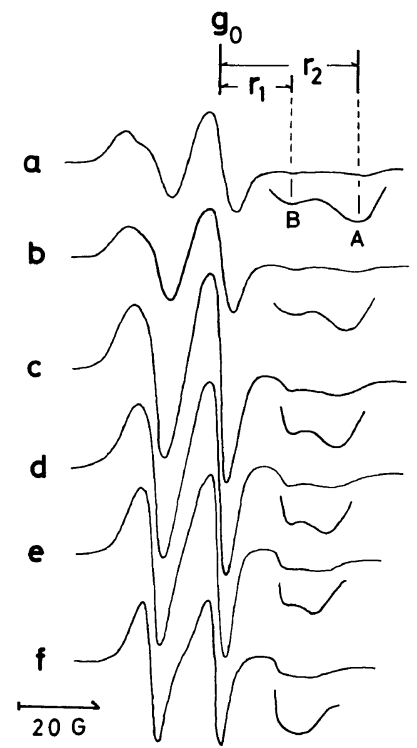

Figure 4. ESR spectra in the temperature region in which the shoulder appears: (a), $138^{\circ} \mathrm{C}$; (b), $149^{\circ} \mathrm{C}$; (c), $158^{\circ} \mathrm{C}$; (d), $166^{\circ} \mathrm{C}$; (e), $177^{\circ} \mathrm{C}$; (f), $188^{\circ} \mathrm{C}$.

non labeled PMMA are shown in Figure 7. The temperature variation of the second moments is tabulated in Table I. The theoretical second moment was calculated for some modes of reorientation possible in PMMA by Sinnot. ${ }^{10}$ For the following two cases - the rotation of both methyl groups with the remainder of the molecule being stationary and the rotation of an entire repeat unit including both methyl groups - the calculated second moments are 12.4 and 4.4 gauss, ${ }^{2}$ respectively. Comparison of our data of the second moment with these theoretical

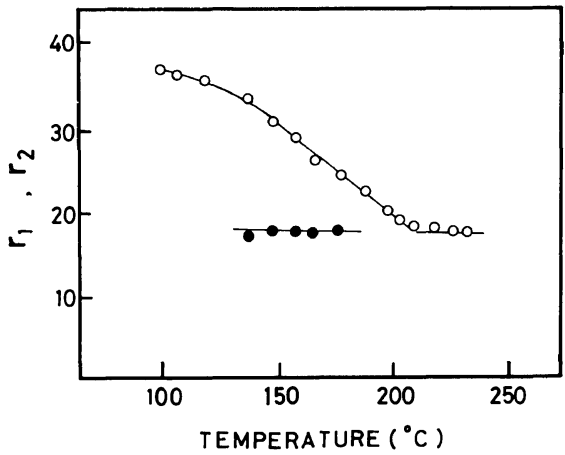

Figure 5. The temperature dependence of the position of the shoulder and the extreme peak: $\bigcirc$, the interval of the isotropic $g$ and the extreme peak $\mathrm{A}\left(r_{2}\right)$; 0 , the interval of the isotropic $g$ and the shoulder B $\left(r_{1}\right)$.

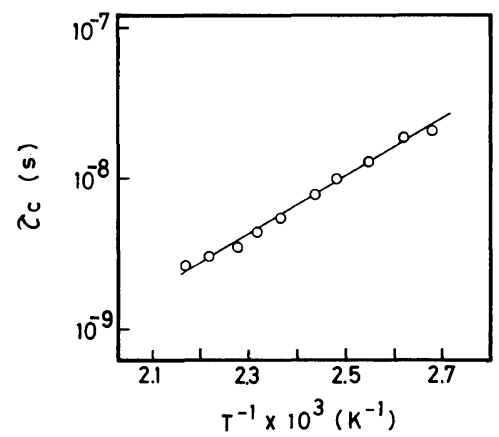

Figure 6. Arrhenius plot of the rotational correlation times below $181^{\circ} \mathrm{C}$.

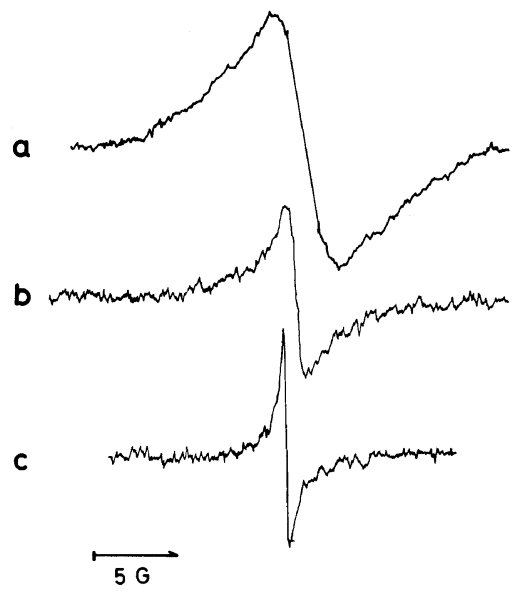

Figure 7. The broad-line ${ }^{1} \mathrm{H}$ NMR spectra at various temperatures: (a), $22^{\circ} \mathrm{C}$; (b), $97^{\circ} \mathrm{C}$; (c), $106^{\circ} \mathrm{C}$.

values indicates that the two methyl groups are 
Table I. Second moments of the ${ }^{1} \mathrm{H}$ NMR spectra at various temperatures

\begin{tabular}{rc}
\hline \multicolumn{1}{c}{$T$} & Second moment \\
\cline { 2 - 2 }${ }^{\circ} \mathrm{C}$ & (gauss) $^{2}$ \\
\hline 22.0 & 11.3 \\
75.5 & 7.6 \\
97.0 & 6.2 \\
106.0 & 3.7 \\
\hline
\end{tabular}

rotating above room temperature and the entire monomer unit rotates sufficiently rapidly at about $100^{\circ} \mathrm{C}$.

\section{DISCUSSION}

It is possible for the shoulder shown in Figure 4 to appear in an ESR spectrum observed for an amorphous sample when the anisotropies of both the $g$ and hyperfine $A$ tensors and the line-width are suitable. In this case, the shoulder is merely an apparent one but has no physical meaning. In our spectra, this possibility is ruled out because of the following two reasons. If this shoulder is the apparent one, its position should move with the temperature change. Since the temperature variation of the peak indicates a partial averaging of the anisotropy of the coupling constant, the position $r_{1}$ of the shoulder B must shift simultaneously. However, in our spectra, the position $r_{2}$ of the extreme peak, A, changes gradually with the temperature change but no shift was observed in the shoulder, as shown in Figure 5. The second reason follows from the position of the shoulder. This position is coincident with the peak position of the spectrum obtained in the case of complete averaging. Accordingly, this shoulder has a real physical meaning and indicates that the spectrum involves a component which is observed from the labeled PMMA rotating rapidly for the complete average of the anisotropic coupling constant. This sample does not have a single tacticity. Thus, it may be possible to attribute these two components in the ESR spectra to the difference in the tacticity. Similar experiments were done for the completely isotactic samples, but no difference in the line-shape was observed for this sample, meaning that there is no correspondence between the fractions of the spectra and the tacticity. The sample is not monodisperse but has some distribution in the molecular weight, as shown in Figure 1a. This fraction might be also the origin of the shoulder B. The fractionated sample, from which the lower molecular weight was cut, also showed the same ESR behaviour in Figure 3, and had the shoulder. Accordingly this fraction of the lower molecular weight has no relation to the ESR lineshape. Since the glass-transition temperature of isotactic PMMA is $45^{\circ} \mathrm{C},{ }^{11}$ the temperature range of the shoulder appearance is considered to be in the rubbery state. However, the second moment of the NMR line is 6.2 gauss ${ }^{2}$ even at $97^{\circ} \mathrm{C}$, and this is larger than the second moment for the freely rotating main chain. This difference in the temperature is attributed to the difference in the time scale for the higher frequency used for NMR; the free rotating main chain detected by NMR shifts to higher temperatures. In the temperature range of Figure 4, one component is gradually averaged, though the temperatures are higher than those for the free rotation in the NMR time-scale. This is again due to the time factor used in ESR. The rate needed for averaging the $A$ anisotropy is usually $10^{10} \mathrm{~s}^{-1}$, which is much higher than the NMR frequency. Thus, in this temperature region, one fraction of PMMA is gradually activated to the higher rate and the other fraction has a sufficiently higher rate for the averaging and shows the isotropic coupling constant appearing as the shoulder even at $138^{\circ} \mathrm{C}$. Therefore, one may conclude from the ESR experiments that there are two kinds of motion at $138^{\circ} \mathrm{C}$; one is sufficiently rapid for the complete averaging at the temperature and the other motion is still slow at $138^{\circ} \mathrm{C}$ and becomes gradually activated with raised temperature. On the other hand, it is known from the mechanical and dielectric dispersion experiments that there are several modes of molecular motion. However, one should not make a simple correspondence between the two kinds of motion detected by ESR to the two processes, $\alpha$ and $\beta$. The onset of the rapid motion of the ester side chain ( $\beta$ process) averages the anisotropy of the nitrogen coupling in the labeled radical at the site of the ester side chain. Similarly, the rapid motion of the segmental chains ( $\alpha$ process) also causes the averaging the h.f. coupling. The temperature region in which the two kinds of motion appear is much higher than the glasstransition temperature, $T_{\mathrm{g}}$, and it is reasonable that the main mode at this temperature region is the micro-Brownian motion of the main chain. The 
Existence of Two Phases in Poly(methyl methacrylate)

above line of thought suggest that the most reasonable origin of the two kinds of motion is the difference in the aggregation state of PMMA molecules, that is, the coexistence of two different phases in the sample. We do not feel that the shoulder $\mathrm{B}$ is due to the presence of crystalline regions in the polymer because $\mathrm{X}$-ray profiles of this sample showed negligible small of crystalline materials.

Acknowledgement. The authors should like to express their gratitude to Dr. F. L. Cummings for his editing of the English in this paper.

\section{REFERENCES}

1. Y. Ishida, S. Togami, and K. Yamafuji, J. Polym. Sci, B, 5, 745 (1967).
2. G. I. Mikhalov and T. I. Borisova, Polym. Sci., USSR, 2, 387 (1961).

3. A. Tanaka and Y. Ishida, J. Polym. Sci., 13, 335 (1974).

4. L. J. Berliner, Ed., "Spin Labelling," Academic Press, New York, N.Y., 1976.

5. A. T. Bullock et al., (a) Eur. Polym. J., 7, 445 (1971); (b) J. Polym. Sci., Polym. Phys. Ed., 11, 1263 (1973).

6. (a) N. Kusumoto, M. Yonezawa, and Y. Motozato, Polymer, 15, 793 (1974); (b) N. Kusumoto, S. Sano, N. Zaitsu, and Y. Motozato, ibid., 17, 448 (1976).

7. S. Shiotani and J. Sohma, Polym. J., 9, 283 (1977).

8. Z. Veksli and W. G. Miller, (a) Macromolecules, 8, 248 (1975); (b), ibid., 10, 686 (1977).

Z. Veksli, W. G. Miller, and E. L. Thomas, J. Polym. Sci., Polym. Symposia, No. 54, 299 (1976).

9. S. A. Goldman, G. V. Bruno, and J. H. Freed, J. Phys. Chem., 76, 1858 (1972).

10. K. M. Sinnot, J. Polym. Sci., 42, 3 (1960).

11. W. E. Goodie, F. H. Owens, R. P. Fellmann, and W. H. Snyder, J. Polym. Sci., 46, 317 (1960). 